\title{
Colorimetric Detection of Copper (II) Based on the Self-Assembly of Schiff's Base-Functionalized Gold Nanoparticles
}

\author{
Yucong Wang ${ }^{1}$, Xiaoli $\mathrm{Li}^{1}$, Yingxue Zhou ${ }^{1} \&$ Chenghui Liu ${ }^{1}$ \\ ${ }^{1}$ College of Chemistry and Environmental Science, Hebei University, Hebei Province, China. \\ Correspondence: Yucong Wang, College of Chemistry and Environmental Science, Hebei University, Baoding \\ 071002, Hebei Province, China. E-mail: Wangyucong@hbu.edu.cn
}

Received: June 25, 2012

Accepted: July 18, 2012 Online Published: July 29, 2012

doi:10.5539/ijc.v4n4p90

URL: http://dx.doi.org/10.5539/ijc.v4n4p90

\begin{abstract}
A simple and highly selective colorimetric sensor for $\mathrm{Cu}^{2+}$ is fabricated in this work based on the $\mathrm{Cu}^{2+}$-induced self-aggregation of gold nanoparticles (GNPs). In this new design, GNPs are first functionalized with cysteamine molecules on their surface through the well-known strong Au-S interaction. Then salicylaldehyde is introduced to link with the cysteamine through a simple Schiff's base reaction. Since the Schiff's bases formed on the GNPs surface may serve as efficient and selective complexing reagents for $\mathrm{Cu}^{2+}, \mathrm{Cu}^{2+}$ can specifically react with the Schiff's base complexes and thus result in the self-assembly of GNPs accompanied with color changes. Therefore, a facile and low-cost colorimetric sensor for visual detection of $\mathrm{Cu}^{2+}$ was developed without requirement of any instrument.
\end{abstract}

Keywords: Gold nanoparticle, colorimetric detection, Copper, self-assembly

\section{Introduction}

Copper is a transition metal which plays important roles in either environmental or biological systems. It is well known that copper is an essential trace element for humans and other animals. However, $\mathrm{Cu}^{2+}$ will become highly toxic to humans at high concentrations (Barranguet et al., 2003). For example, some cases of liver damage of children have been proved to be associated with the excessive intake of copper ions (Zietz et al., 2003). Furthermore, high concentrations of copper will also destroy the biological reprocessing systems in water (Zhao et al., 2009). Therefore, the fabrication of sensitive and selective methods to monitor copper ions in either environmental samples or biological samples is of great significance.

Up to now, several methods for $\mathrm{Cu}^{2+}$ detection have been reported, including organic fluorophore-based assays (Viguier et al., 2006; Xiang et al., 2006), atomic absorption spectroscopy (AAS) (Gonzales et al., 2009), inductively coupled plasma mass spectroscopy (ICP-MS) (Becker et al., 2005), ICP-AES (atomic emission spectroscopy) (Liu et al., 2005) and some electrochemical assays (Ensafi et al., 2006; Karimi et al., 2006). Although the sensitivities of these developed methods for $\mathrm{Cu}^{2+}$ are generally high, most of these assays need either expensive and speciallized instruments or sophisticated assay procedures, which greatly limited their wide applications. Therefore, it is still imperative to develop simple and efficient $\mathrm{Cu}^{2+}$ sensor especially for field work or point of care testing of $\mathrm{Cu}^{2+}$.

In recent years, gold nanoparticles (GNPs) are becoming increasingly attractive in the fabrication of optical sensors, devices as well as in biodiagnostics and medical therapeutics due to their excellent chemical and physical properties (Rosi et al., 2005; Lu et al., 2007). They have strong surface plasmon resonance (SPR) absorption in the visible wavelength range with extremely high extinction coefficients. One attractive property of GNPs is that their SPR frequency will be changed dramatically when the distance between GNPs varied. Therefore, the analyte-induced aggregation of GNPs offered a facile colorimetric approach for the simple visual detection of nucleic acids (Elghanian et al., 1997; Li et al., 2004), proteins (Zhen et al., 2012), small molecules (Jiang et al., 2010; Wang et al., 2007), and metal ions (Chen et al., 2009).

Inspired by these elegantly designed GNPs-based colorimetric saasys, we wish to report in this contribution a simple and highly specific colorimetric method for $\mathrm{Cu}^{2+}$ detection based on the $\mathrm{Cu}^{2+}$-induced self-aggregation of GNPs. In this work, GNPs were first functionalized with cysteamine (CA) through Au-S bond. Then a simple Schiff's base formation reaction was carried out on the GNPs surface between the amine group of CA and 
aldehyde group of salicylaldehyde (SAL). The Schiff's bases formed on the GNPs surface may serve as efficient and selective complexing reagents for $\mathrm{Cu}^{2+}$ (Karimi et al., 2006). Therefore, the GNPs-Schiff base complexes can be applied as a facile and low-cost colorimetric sensor for visual detection of $\mathrm{Cu}^{2+}$ without requirement of any instrument, which is particularly promissing in the applications for field work or point of care testing of $\mathrm{Cu}^{2+}$.

\section{Experimental Section}

\subsection{Reagents}

Tetrachloroauric acid $\left(\mathrm{HAuCl}_{4} \cdot 4 \mathrm{H}_{2} \mathrm{O}\right)$ was obtained from Sinopharm Group. Cysteamine and salicylaldehyde was purchased form Sigma-Aldrich. All other chemicals were obtained from Beijing Chemical Corp., all of which were of analytical grade and used as received without further purification.

\subsection{Preparation of GNPS}

GNPs were prepared by citrate reduction of $\mathrm{HAuCl}_{4}$ according to Frens (Frens, 1973) and Grabar (Grabar et al., 1995 ) et al. with slight modification. All glassware were soaked in aqua regia and carefully cleaned before use. Briefly, after boiling a $100 \mathrm{~mL}$ of $0.01 \% \mathrm{HAuCl}_{4}$ solution, $3.5 \mathrm{~mL}$ of the $1.0 \%$ trisodium citrate solution was quickly added with vigorous stirring. The color of the solution changed to deep red in a few seconds and the boiling was continued for $10 \mathrm{~min}$. After the solution was naturally cooled to room temperature, it was filtered through a $0.22-\mu \mathrm{m}$ membrane filter and diluted to $100.00 \mathrm{~mL}$. The average diameter of the GNPs is $16 \mathrm{~nm}$.

\subsection{Preparation of GNPS-CA-SAL Complexes}

Typically, $100 \mu \mathrm{L}$ of $10^{-5} \mathrm{M} \mathrm{CA}$ was added into $4 \mathrm{~mL}$ of GNPs drop by drop with shaking. After incubation at $4{ }^{\circ} \mathrm{C}$ for $2 \mathrm{~h}, 120 \mu \mathrm{L}$ of ethanol solution containing $10^{-5} \mathrm{M} \mathrm{SAL}$ was slowly added into the GNPs with shaking to allow the formation of Schiff' bases on the GNPs surface. After standing for $4 \mathrm{~h}$ at $4{ }^{\circ} \mathrm{C}$, the mixture was centrifuged at 12,000 rpm for 20 min to remove unbound CA and ALS molecules. The precipitated red oily GNPs-CA-ALS complexes were then washed twice and finally dispersed in $4 \mathrm{ml}$ water.

\subsection{Standard Detection Procedures for $\mathrm{Cu}^{2+}$}

Briefly, $0.36 \mathrm{~mL}$ of the as-prepared GNPs-CA-SAL complexes was put into a $0.5 \mathrm{~mL}$ Eppendorf tube. Then 40 $\mu \mathrm{L}$ of $\mathrm{Cu}^{2+}$ solution with certain concentration was transferred in and mixed thoroughly with a vortex mixer. After incubation at room temperature for $1 \mathrm{~h}, \mathrm{UV} / \mathrm{Vis}$ absorption measurements were conducted using a TU-1901 UV/Vis Spectrophotometer (Purkinje General) and the photos were taken within 5 min.

\section{Results and Discussion}

\subsection{Design Principle of the Proposed Approach}

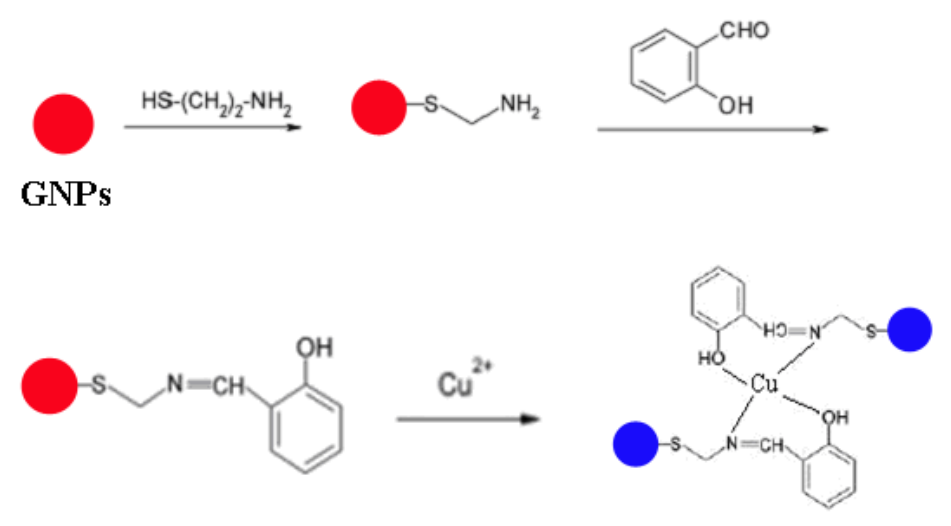

Figure 1. Schemetic representation of the proposed colorimetric $\mathrm{Cu}^{2+}$ sensor

This assay utilizes the GNPs assembly-induced changes in color as well as UV-Vis spectrometry. Figure 1 outlines the general principle of the sensing strategy for visual screening of $\mathrm{Cu}^{2+}$ in this work. As shown in Figure 1, CA was first immobilized on the GNPs surface through Au-S covalent bond. Then SAL was covalently linked with CA to form a Schiff's base. The Schiff' bases-functionalized GNPs were red in color and exhibit a characteristic absorption at $520 \mathrm{~nm}$. The Schiff's base of SAL and CA was efficient complexing agent for $\mathrm{Cu}^{2+}$. Therefore, upon the addition of $\mathrm{Cu}^{2+}, \mathrm{Cu}^{2+}$ will react with the Schiff' bases on the surfaces of different GNPs. As a result, GNPs would assemble with each other via $\mathrm{Cu}^{2+}$ to form a net-work structure, which was accompanied 
with changes in color (from red to purple and blue) and in the UV-Vis spectrum of GNPs, with the decrease of absorption at $520 \mathrm{~nm}$ and a new absorption at $620 \mathrm{~nm}$. Consequently, the resulting color change and absorption spectral response of GNPs can then give an indicator for the presence of $\mathrm{Cu}^{2+}$.

\subsection{Analytical Performance}

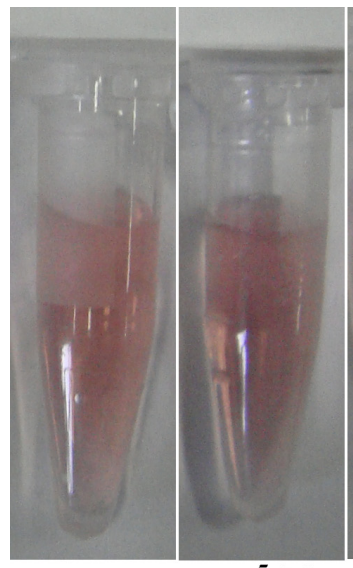

Blank

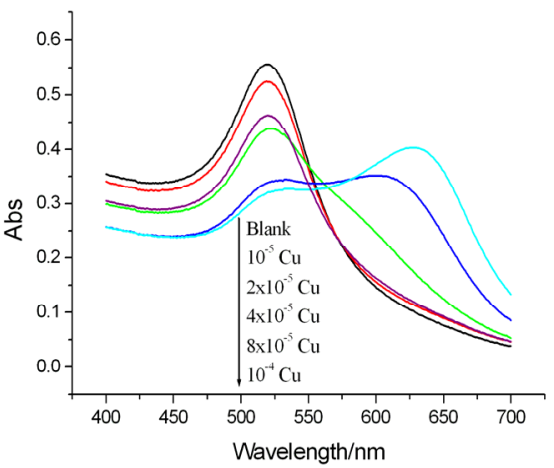

(b)

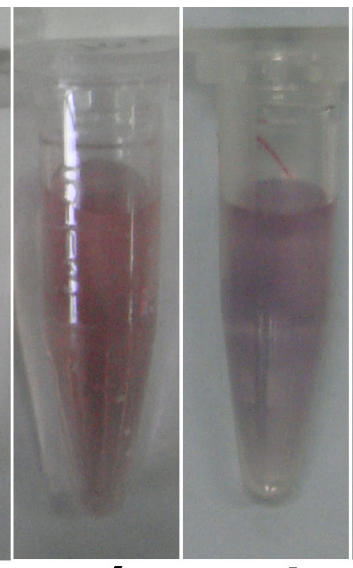

$2 \times 10^{-5} \mathrm{M} 4 \times 10^{-5} \mathrm{M} 8 \times 10^{-5} \mathrm{M}$

(a)
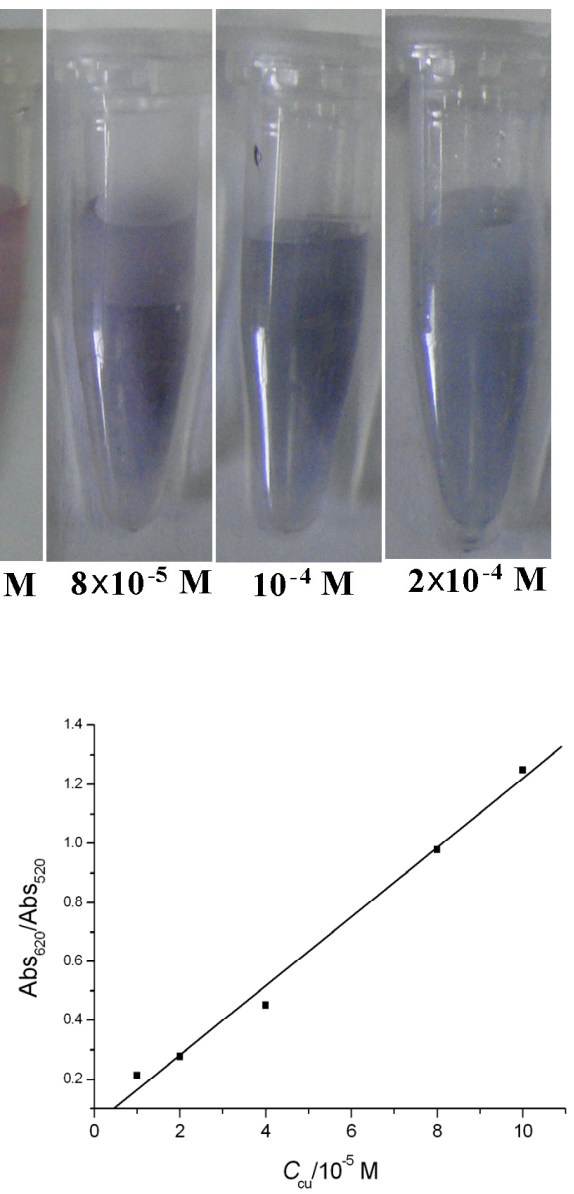

(c)

Figure 2. Analytical performance of the proposed colorimetric assay for $\mathrm{Cu}^{2+}$. (a) Photographs and (b) UV-Vis spectra of Schiff' bases-functionalized GNPs dispersions by addition of different concentrations of $\mathrm{Cu}^{2+}$. (c) Plot of $\mathrm{Abs}_{620} / \mathrm{Abs}_{520} \mathrm{vs} \mathrm{Cu}^{2+}$ concentrations for the quantitative detection of $\mathrm{Cu}^{2+}$

To evaluate the analytical performance of the proposed sensor, different concentrations of $\mathrm{Cu}^{2+}$ were added into individual Schiff' bases-functionalized GNPs, and the color change and UV-Vis spectra were recorded. As shown in Figure 2a, upon the addition of $\mathrm{Cu}^{2+}$, the aggregation of GNPs occurred, resulting in the color variation from red to purple and then blue with the increase of $\mathrm{Cu}^{2+}$ concentrations. This aggregation process can be monitored visually, thus the naked eye alone can judge the presence of $\mathrm{Cu}^{2+}$ ions without the requirement of any instruments. One can see from Figure 2a that only when the $\mathrm{Cu}^{2+}$ concentration was higher than $10^{-5} \mathrm{~m}$, the changes of color could be observed obviously. That is to say, the minimum concentration of $\mathrm{Cu}^{2+}$ detectable by eye is approximately $10^{-5} \mathrm{~m}$. Although this lowest detectable concentration cannot be compared with those obtained with advanced instruments, however, it should be noted that this proposed assay is believed to be the most sensitive for the detection of $\mathrm{Cu}^{2+}$ ions by the naked eye alone (Zhou et al., 2008).

The UV-Vis absorption spectra (Figure $2 b$ ) further demonstrate the aggregation of GNPs and the color changes induced by the addition of $\mathrm{Cu}^{2+}$. The pure GNPs-CA-SAL dispersion shows an absorption peak at $520 \mathrm{~nm}$. However, increasing the concentration of $\mathrm{Cu}^{2+}$ resulted in a decrease in the absorbance at $520 \mathrm{~nm}\left(\mathrm{Abs}_{520}\right)$ and a clear increase in the absorbance at $625 \mathrm{~nm}\left(\mathrm{Abs}_{625}\right)$, which was ascribed to the absorbance of the aggregated GNPs. The ratio of $\mathrm{Abs}_{625}$ to $\mathrm{Abs}_{520}$ was found to be linear with the $\mathrm{Cu}^{2+}$ concentration within the concentration range from $10^{-5} \mathrm{~m}$ to $10^{-4} \mathrm{~m}$ (Figure $2 \mathrm{c}$ ) with the regression equation $\mathrm{y}=0.12 \mathrm{x}+0.05(\mathrm{R}=0.9953)$. 


\subsection{Specificitry Evaluation}

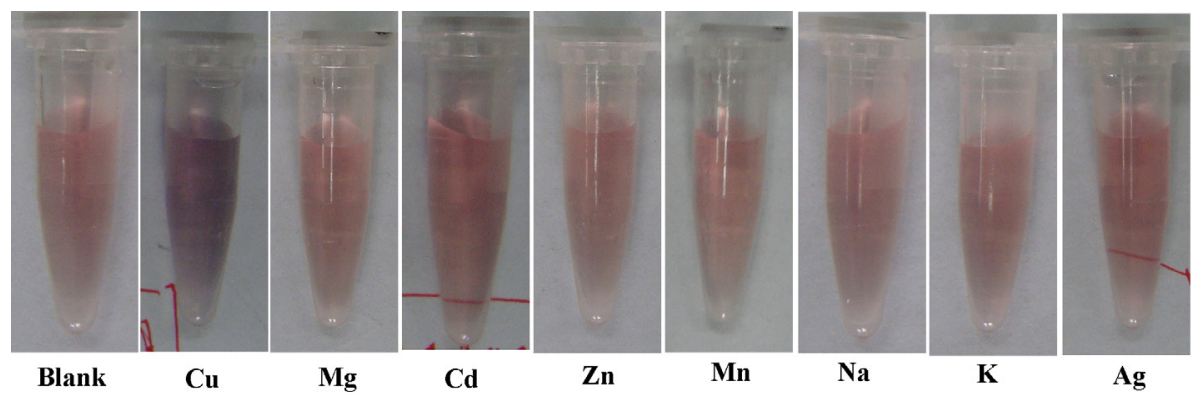

(a)

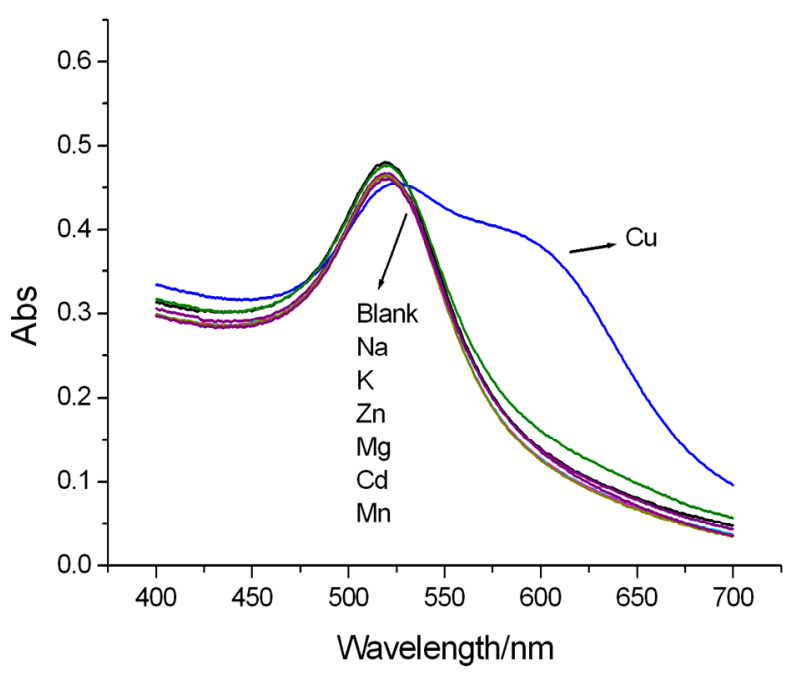

(b)

Figure 3. Specifity evaluation of the proposed assay for $\mathrm{Cu}^{2+}$ against other metal ions. (a) Photographs and (b) UV-Vis spectra of GNPs-CA-SAL dispersions by addition of different metal ions. The concentrations of these metal ions were all kept at $5 \times 10^{-5} \mathrm{~m}$

We tested the specificiy of this assay for $\mathrm{Cu}^{2+}$ ions by using other metal ions in place of $\mathrm{Cu}^{2+}$, including the frequently used $\mathrm{Mg}^{2+}, \mathrm{Cd}^{2+}, \mathrm{Zn}^{2+}, \mathrm{Mn}^{2+}, \mathrm{Na}^{+}, \mathrm{K}^{+}$and $\mathrm{Ag}^{+}$with the concentrations kept at $5 \times 10^{-5} \mathrm{M}$. As can be seen from Figure 3, only the $\mathrm{Cu}^{2+}$ ions could induce significant color change from red to purple and obvious UV-Vis absorption change. No distinct color changes or UV-Vis absorbance variations were observed for other metal ions. These results clearly show that the specificity of the proposed assay is extremely high for $\mathrm{Cu}^{2+}$ detection and none of other metal ions interfered with the assay.

\section{Conclusion}

In conclusion, we have developed a simple colorimetric sensor for visual detection of $\mathrm{Cu}^{2+}$ by using Schiff' bases-functionalized GNPs. Most attractively, this method is rapid, convenient and low-cost because no instruments are required and the presence of $\mathrm{Cu}^{2+}$ can be easily monitored by the naked eyes. Although the sensitivity of the proposed visual detection method is not yet comparable with those methods performed on advanced instruments, this assay has been among the most sensitive ones for the detection of $\mathrm{Cu}^{2+}$ ions by the naked eye alone. Extremely high assay specificity for $\mathrm{Cu}^{2+}$ is another striking advantage of this proposed method since most of the frequently used metal ions do not interfere with the detection of $\mathrm{Cu}^{2+}$ at all. We believe that this method may find wide applications in many fields such as in the environmental or biological systems where $\mathrm{Cu}^{2+}$ monitoring is required. Without the need for advance equipments that is typically bulky and expensive, the proposed approach is extremely suitable for fabricating miniatured and portable sensor, which is extremely promissing for the applications out of the laboratory. 


\section{Acknowledgements}

This work was supported by the National Natural Science Foundation of China (20905018), the Natural Science Foundation of Hebei Province (B2012201108, B2010000202), the S\&T plan project of Hebei Provincial Education Department (2009306) and China Postdoctoral Science Foundation (20090450920).

\section{References}

Barranguet, C., van den Ende, F. P., Rutgers, M., Breure, A. M., Greijdanus, M., Sinke, J. J., \& Admiraal, W. (2003). Copper-induced modifications of the trophic relations in riverine algal-bacterial biofilms. Environ. Toxicol. Chem., 22, 1340-1349. http://dx.doi.org/10.1002/etc.5620220622

Becker, J. S., Zoriy, M. V., Pickhardt, C., Palomero-Gallagher, N., \& Zilles, K. (2005). Quantitative imaging of selenium, copper, and zinc in thin sections of biological tissues (slugs-genus arion) measured by laser ablation inductively coupled plasma mass spectrometry. Anal. Chem., 77, 6074-6080. http://dx.doi.org/10.1021/ac0700528

Chen, Y. Y., Chang, H. T., Shiang, Y. C., Hung, Y. L., Chiang, C. K., \& Huang, C. C. (2009). Colorimetric Assay for Lead Ions Based on the Leaching of Gold Nanoparticles. Anal. Chem., 81, 9433-9439. http://dx.doi.org/10.1021/ac9018268

Elghanian, R., Storhoff, J. J., Mucic, R. C., Letsinger, R. L. \& Mirkin, C. A.(1997). Selective Colorimetric Detection of Polynucleotides Based on the Distance-Dependent Optical Properties of Gold Nanoparticles. Science, 277, 1078-1081. http://dx.doi.org/10.1126/science.277.5329.1078

Ensafi, A. A., Khayamian, T., \& Benvidi, A. (2006). Simultaneous determination of copper, lead and cadmium by cathodic adsorptive stripping voltammetry using artificial neural network. Anal. Chim. Acta, 561, 225-232. http://dx.doi.org/10.1016/j.aca.2006.01.015

Frens, G. (1973). Controlled nucleation for the regulation of the particle size in monodisperse gold suspensions. Nat. Phys. Sci., 241, 20-22. http://dx.doi.org/10.1038/physci241020a0

Grabar, K. C., Freeman, R. G., Hommer, M. B., \& Natan, M. J. (1995). Preparation and Characterization of Au colloid Monolayers. Anal. Chem., 67, 735-743. http://dx.doi.org/10.1021/ac00100a008

Gonzales, A. P. S., Firmino, M. A., Nomura, C. S., Rocha, F. R. P., Oliveira, P. V., \& Gaubeur, I. (2009). Peat as a natural solid-phase for copper preconcentration and determination in a multicommuted flow system coupled to flame atomic absorption spectrometry. Anal. Chim. Acta, 636, 198-204. http://dx.doi.org/10.1016/j.aca.2009.01.047

Jiang, Y., Zhao, H., Lin, Y., Zhu, N., Ma, Y., \& Mao, L. (2010). Colorimetric Detection of Glucose in Rat Brain Using Gold Nanoparticles. Angew. Chem. Int. Ed., 49, 4800-4804. http://dx.doi.org/10.1002/anie.201001057

Karimi Shervedani R., \& Ahmad Mozaffari S. (2006). Copper(II) Nanosensor Based on a Gold Cysteamine Self-Assembled Monolayer Functionalized with Salicylaldehyde. Anal. Chem., 78, 4957-4963. http://dx.doi.org/ 10.1021/ac052292y

Li, H., \& Rothberg, L. (2004). Colorimetric detection of DNA sequences based on electrostatic interactions with unmodified gold nanoparticles. PNAS, 101, 14036-14039. http://dx.doi.org/ 10.1073/pnas.0406115101

Liu, Y., Liang, P., \& Guo, L. (2005). Nanometertitaniumdioxideimmobilized on silicagel as sorbent for preconcentration of metalionsprior to their determination by inductively coupled plasma atom ice mission spectrometry. Talanta, 68, 25-30. http://dx.doi.org/10.1016/j.talanta.2005.04.035

Lu, Y., \& Liu, J. (2007). Smart Nanomaterials Inspired by Biology: Dynamic Assembly of Error-Free Nanomaterials in Response to Multiple Chemical and Biological Stimuli. Acc. Chem. Res., 40, 315-323. http://dx.doi.org/10.1021/ar600053g

Rosi, N. L., \& Mirkin, C. A. (2005). Nanostructures in Biodiagnostics. Chem. Rev., 105, 1547-1562. http://dx.doi.org/10.1021/cr030067f

Viguier, R. F. H., \& Hulme, A. N. (2006). A Sensitized Europium Complex Generated by Micromolar Concentrations of Copper(I): Toward the Detection of Copper(I) in Biology. J. Am. Chem. Soc., 128, 11370-11371. http://dx.doi.org/10.1021/ja064232v

Wang, J., Wang, L., Liu, X., Liang, Z., Song, S., Li, W., Li, G., \& Fan, C. (2007). A Gold Nanoparticle-Based Aptamer Target Binding Readout for ATP Assay. Adv. Mater, 19, 3943-3946. 
http://dx.doi.org/10.1002/adma.200602256

Xiang, Y., Tong, A., Jin, P., \& Ju, Y. (2006). New Fluorescent Rhodamine Hydrazone Chemosensor for Cu(II) with High Selectivity and Sensitivity. Org. Lett., 8, 2863-2866. http://dx.doi.org/10.1021/o10610340

Zhao, Y., Zhang, X. B., Han, Z. X., Li, C. Y., Jian, L. X., Shen, G. L., \& Yu, R. Q. (2009). Highly Sensitive and Selective Colorimetric and Off-On Fluorescent Chemosensor for $\mathrm{Cu}^{2+}$ in Aqueous Solution and Living Cells. Anal. Chem., 81, 7022-7030. http://dx.doi.org/10.1021/ac901127n

Zhen, Z., Tang, L. J., Long H. X., \& Jiang J. H. (2012). Enzymatic Immuno-Assembly of Gold Nanoparticles for Visualized Activity Screening of Histone-Modifying Enzymes. Anal. Chem., 84, 3614-3620. http://dx.doi.org/10.1021/ ac203385v

Zhou, Y., Wang, S., Zhang K., \& Jiang, X. (2008). Visual Detection of Copper(II) by Azide- and Alkyne-Functionalized Gold Nanoparticles Using Click Chemistry. Angew. Chem. Int. Ed., 120, 7564-7566. http://dx.doi.org/10.1002/anie.200802317

Zietz, B. P., Dieter, H. H., Lakomek, M., Schneider, H., Kebler-Gaedtke, B., \& Dunkelberg, H. (2003). Epidemiological investigation on chronic copper toxicity to children exposed via the public drinking water supply. Sci. Total Environ., 302, 127-144. http://dx.doi.org/10.1016/S0048-9697(02)00399-6 